\title{
MORBIDADE E MORTALIDADE HOSPITALAR DE CRIANÇAS MENORES DE UM ANO, EM RIBEIRÃO PRETO, SP (BRASIL), 1975
}

Marilisa Berti de Azevedo Barros*

BARROS, M.B. de A. Murbidade e mortalidade hospitalar de crianças menores de um ano, em Ribeirão Preto, SP (Brasil), 1975. Rev. Saúde públ., S. Paulo, 15: 308-20, 1981.

RESUMO: Foi estudada a morbi-mortalidade hospitalar de crianças menores de um ano, residentes em Ribeirão Preto, SP (Brasil), internadas nos hospitais gerais e no Pronto Socorro Infantil desse município, em 1975. Detectou-se um elevado coeficiente de internação para essa faixa etária $(437 / 1000)$ sendo maior no sexo masculino. Apenas três diagnósticos-diarréia, desidratação e pneumonia - foram responsáveis por $80,36 \%$ das internações. A morbidade hospitalar apresentou diferenças segundo a categoria de internação das crianças. Observou-se que $75 \%$ dos obitos hospitalares foram decorrentes de doenças infecciosas. A letalidade hospitalar foi 3 vezes mais elevada nas crianças "indigentes" do que naquelas de categoria particular.

UNITERMOS: Hospital. Mortalidade. Morbidade. Crianças hospitalizadas, Ribeirão Preto, SP Brasil.

\section{INTRODUCAO}

É amplamente reconhecido o maior risco de mortalidade a que as pessoas estão expostas em seu primeiro ano de vida. É justamente a maior suscetibilidade que os lactentes apresentam às condiçōes ambientais que tem conferido ao coeficiente de mortalidade infantil (CMI) o papel de indicador não apenas de saúde mas, inclusive, do "nivel de vida" da população '

A maior taxa de óbitos das crianças nesta faixa etária resulta da maior incidência de doenças associada à menor resistência orgânica. Assim como a mortalidade ${ }^{11}$, a morbidade infantil é de maior incidência nas áreas subdesenvolvidas e a sua distribuição se faz desigualmente nos diferentes estratos sócio-econômicos da população.

A forma como os diferentes grupos sociais se inserem na estrutura de produção e consequientemente na participação da riqueza produzida, lhes confere não apenas um perfil diferenciado de morbidade, mas ainda um acesso discriminado aos serviços de assistência médica.

O contingente de crianças menores de um ano deverá ter uma presença importante na utilização de serviços de saúde, apresentando um padrão de doenças diferente segundo o segmento sócio-econômico do qual procede.

* Do Departamento de Medicina Preventiva e Social da Faculdade de Ciências Médicas da UNICAMP - Rua Dr. Quirino, 1856 - 13100 - Campinas, SP - Brasil. 
BARROS, M.B. de A. Morbidade e mortalidade hospitalar de crianças menores de um ano, em Ribeirão Preto, SP (Brasil), 1975. Rev. Saúde puibl., S. Paulo, 15:308-20, 1981.

Quanto aos serviços de atenção médica no Brasil, verifica-se que, em linhas gerais, as instituições prestadoras de cuidados à saúde caracterizam-se pela atuação descoordenada, pelo privilegiamento do setor privado e por uma atenção médica predom:nantemente hospitalar. E flagrante a disparidade dos gastos dispendidos no atendimento hospitalar frente ao realizado com a assistência ambulatorial e de saúde pública ".

Embora a população infantil venha sendo alvo de programas que visam basicamente à prevenção primária, é preciso acompanhar - impacto dessas ações a nivel da morbidade hospitalar das crianças.

O propósito deste estudo, que se baseia em dados que fazem parte de um trabalho mais amplo ', é o de analisar a morbimortalidade hospitalar de crianças com menos de 1 ano, identificando os problemas de maior magnitude neste nivel de serviços e buscando detectar diferenciações no perfil de morbidade, entre as categorias de internação dos pacientes.

\section{MATERIAL E METODOS}

Foram estudadas as altas hospitalares de crianças menores de um ano, residentes em Ribeirão Preto, internadas nos hospitais gerais e no Pronto Socorro Infantil do municipio, no decorrer de 1975.

As informações relativas às altas foram coletadas e processadas pelo Centro de Processamento de Dados Hospitalares ( $\mathrm{CPDH})$, vinculado ao Departamento de Medicina Social da Faculdade de Medicina de Ribeirão Preto. A estrutura e funcionamento do CPDH foram descritas e analisadas por Fávero e col.3 (1973) e Yazlle Rocha is (1975).

Foram incluidas neste estudo apenas as crianças internadas no hospital por efeito de alguma patologia e não os recém-nascidos que ali se encontravam em decorrência de parto-hospitalar.

A respeito de cada egresso foram estuóadas as seguintes variáveis: sexo, idade, local de residência, categoria de internação, diagnóstico principal e nos casos que foram a óbito, o diagnóstico correspondente.

Os diagnósticos foram codificados e classificados segundo os 17 grupos e a lista A da Classificação Internacional das Doenças (CID) em sua 8 " revisão *

A qualidade do diagnóstico é assegurada por se tratar de um diagnóstico hospitalar e ser registrado na ficha utilizada pelo $\mathrm{CPDH}$, no momento de alta do paciente, cuando já foram ou não confirmadas as hipóteses diagnósticas efetuadas por ocasião da internação.

Quanto à categoria de internação, os pacientes foram classificados em: particular - quando a hospitalização foi paga por familiares da criança; indigente - quando foi gratuito o atendimento médico-hospitalar; INPS (Instituto Nacional de Previdência Social) ${ }^{*}$ - quando a internação foi custeada por esta instituição previdenciária; outro seguro - que incluiu as hospitalizaçōes custeadas pelas demais instituições como UNIMED, IAMSPE, entre outras.

A categoria de internação, ao mesmo tempo em que representa as instituições médicas a que as pessoas podem ter acesso, indicando, de modo imediato, tipos diferentes de serviços de saúde com formas peculiares de funcionamento, pode também ser tomada como um indicador de categoria sócio-econômica, dado que é o tipo de inserção das pessoas no mercado de trabalho que define a modalidade de assistência médica que podem obter. Foi verificada, em trabalho anterior ${ }^{1}$, uma diferenciação sócio-

* Atual Instituto Nacional de Assistência Médica e Previdêncta Social (INAMPS). 
BARROS, M.B. de A. Morbidade e mortalidade hospitalar de crianças menores de um ano, em Ribeirão Preto, SP (Brasil), 1975. Rev. Saúde públ., S. Paulo, 15:308-20, 1981

-ocupacional em função da categoria de internação do paciente.

Em virtude dos numerosos recursos para a saúde existentes em Ribeirão Preto, que se configura inclusive, como um centro regional de assistência médica, é possivel assegurar como Yazlle Rocha ${ }^{17}$ (1975) que a quase totalidade do atendimento hospitalar dos moradores do município se dá localmente.

\section{RESULTADOS E COMENTÁRIOS}

\section{Coeficientes de Internação}

A hospitalização de crianças menores de um ano de idade, revelou-se bastante elerada, atingindo 437 internaçōes para cada 1.000 crianças dessa faixa etária; este valor é três vezes superior ao coeficiente médio da população do município de Ribeirão Preto, em 1975 (Tabela 1).

T A B E L A 1

Internações totais e de menores de um ano, de residentes em Ribeirão Preto. segundo o sexo, em 1975.

\begin{tabular}{|c|c|c|c|c|}
\hline Sexo & Idade & $\begin{array}{l}\text { Número de } \\
\text { Internações }\end{array}$ & $\begin{array}{c}\text { População estimada } \\
(1 / 7 / 1975)\end{array}$ & $\begin{array}{l}\text { Coef. de internação } \\
\text { (por } 1.000 \text { hab.) }\end{array}$ \\
\hline \multirow{2}{*}{ Masculino } & Menores de 1 ano & 1.292 & 2.702 & 478,16 \\
\hline & Todas as idades & 12.713 & 120.689 & 105,34 \\
\hline \multirow{2}{*}{ Feminino } & Menores de 1 ano & 1.035 & 2.622 & 394,74 \\
\hline & Todas as idades & 19.938 & 126.790 & 157,25 \\
\hline \multirow{2}{*}{ Total } & Menores de 1 ano & 2.327 & 5.324 & 437,08 \\
\hline & Todas as idades* & 32.651 & 247.479 & 131,93 \\
\hline
\end{tabular}

* Excluídas 26 altas com informações prejudicadas.

Para uma comparação do coeficiente de internação verificado em Ribeirão Preto, pode-se tomar o encontrado nos EUA, para o mesmo periodo de tempo, que foi de 209 internaçóes para cada mil crianças menores de um ano ${ }^{*}$; é preciso lembrar que este país apresenta as maiores taxas de internação quando comparado a países europeus $5: 14$ e ainda assim as crianças de $\mathrm{Ri}$ beirão Preto apresentam taxa duas vezes maior que a das crianças dos EUA.

$O$ coeficiente de internação foi maior nas crianças do sexo masculino, enquanto que a taxa global (para todas as idades) é mais elevada no feminino.
É fenômeno conhecido a maior frequêencia de doenças e de óbitos nas crianças do sexo masculino, inclusive, as perdas fetais atingem este sexo com mais intensidade. Nas outras idades, entretanto, é o sexo feminino que refere mais morbidade e utiliza mais intensamente serviços médicos, embora continue apresentando as menores taxas de mortalidade.

As internações de crianças menores de um ano representaram $7,13 \%$ do total das altas de residentes em Ribeirão Preto, ocorridas em 1975, sendo notável a diferença deste percentual entre os sexos (Tabela 2). 
BARROS, M.B. de A. Morbidade e mortalidade hospitalar de crianças menores de um ano, em Ribeirão Preto, SP (Brasil), 1975. Rev. Saúde públ., S. Paulo, 15:308-20, 1981.

T A B E L A 2

Internações de menores de um ano, de residentes em Ribeirão Preto, segundo a categoria de internação e o sexo do paciente, 1975.

\begin{tabular}{|c|c|c|c|c|}
\hline $\begin{array}{c}\text { Categoria de } \\
\text { internação }\end{array}$ & Sexo & $\begin{array}{c}\text { Internação de } \\
<1 \text { ano }\end{array}$ & $\begin{array}{l}\text { Total de } \\
\text { internação }\end{array}$ & $\begin{array}{c}\text { fndice de } \\
\text { internação de } \\
<1 \text { ano }(\%)\end{array}$ \\
\hline Particular & $\begin{array}{l}\mathrm{M} \\
\mathrm{F} \\
\mathrm{T}\end{array}$ & $\begin{array}{l}35 \\
31 \\
66\end{array}$ & $\begin{array}{r}829 \\
1.211 \\
2.040\end{array}$ & $\begin{array}{l}4,22 \\
2,56 \\
3,23\end{array}$ \\
\hline Indigente & $\begin{array}{l}\mathrm{M} \\
\mathrm{F} \\
\mathrm{T}\end{array}$ & $\begin{array}{l}352 \\
275 \\
627\end{array}$ & $\begin{array}{l}2.265 \\
4.934 \\
7.199\end{array}$ & $\begin{array}{r}15,54 \\
5,57 \\
8,71\end{array}$ \\
\hline INPS & $\begin{array}{l}M \\
F \\
T\end{array}$ & $\begin{array}{r}867 \\
683 \\
1.550\end{array}$ & $\begin{array}{r}8.554 \\
12.157 \\
20.711\end{array}$ & $\begin{array}{r}10,13 \\
5,62 \\
7,48\end{array}$ \\
\hline Outros seguros & $\begin{array}{l}\mathrm{MI} \\
\mathrm{F} \\
\mathrm{T}\end{array}$ & $\begin{array}{l}38 \\
46 \\
84\end{array}$ & $\begin{array}{l}1.065 \\
1.636 \\
2.701\end{array}$ & $\begin{array}{l}3.57 \\
2.81 \\
3.11\end{array}$ \\
\hline Total & $\begin{array}{l}M \\
F \\
T\end{array}$ & $\begin{array}{l}1.292 \\
1.035 \\
2.327\end{array}$ & $\begin{array}{l}12.713 \\
19.938 \\
32.651\end{array}$ & $\begin{array}{r}10,16 \\
5,19 \\
7,13\end{array}$ \\
\hline
\end{tabular}

Para esta diferença contribuem os fatos assinalados acima: maior morbidade nas crianças do sexo masculino e maior taxa de uso de serviços pelo sexo feminino, nas demais idades.

Do total de hospitalizações ocorridas para cada categoria de internação, no INPS e na categoria indigente as proporções de internações de crianças revelaram-se mais elevadas, $7,48 \%$ e $8,72 \%$, respectivamente. Poderiam contribuir para esta ocorrência algumas circunstâncias: composição etária mais jovem, maior morbidade infantil e menor taxa de utilização de serviços em outras idades.

A elevada taxa de internação de crianças está a indicar a presença de maior morbidade na população infantil desta cidade que naquelas de países desenvolvidos. Entretanto, num pais subdesenvolvido, taxas tão elevadas só podem ocorrer se existirem leitos pediátricos suficientes e disponiveis, o que se verifica neste município, particularmente.

Torna-se necessário avaliar a adequação destas internações, observando-se os diagnósticos hospitalares destas crianças, como será feito a seguir.

\section{Morbidade Hospitalar}

Os grupos de diagnósticos mais frequientes nas internaçōes de menores de um ano foram: sintomas e estados mórbidos mal-definidos (Grupo XVI), doenças do aparelho respiratório (Grupo VIII) e doenças infectoparasitárias (Grupo I) que, em conjunto, corresponderam a $91,0 \%$ das internações desta faixa etária e a $65 \%$ das internações de todas as idades (Tabela 3). 
BARROS, M.B. de A. Morbidade e mortalidade hospitalar de crianças menores de um ano, em Ribeirão Preto, SP (Brasil), 1975. Rev. Saüie públ., S. Paulo, 15:308-20, 1981.

T A B E L A 3

Diagnósticos das internaçóes de menores de um ano, residentes em Ribeirão Preto, segundo os 17 grupos da Classificação Internacional de Doenças (8a revisão) em 1975.

\begin{tabular}{|c|c|c|c|}
\hline Diagnósticos & No & $\%$ & Coef. $/ 1.000$ \\
\hline I - D. Infecciosas e Parasitárias & 233 & 10,01 & 43,76 \\
\hline II - Tumores & 9 & 0,39 & 1,69 \\
\hline III - D. Gland. Endócrinas, da Nutrição e do Metab. & 19 & 0,82 & 3,57 \\
\hline IV - D. do Sangue e Org. Hematopoéticos & 2 & 0,09 & 0,37 \\
\hline VI - D. do Sist. Nervoso e órgãos dos Sentidos & 35 & 1,50 & 6,57 \\
\hline VII - D. do Aparelho Circulatório & 2 & 0,09 & 0,37 \\
\hline VIII - D. do Aparelho Respiratório & 690 & 29,65 & 129,60 \\
\hline IX - D. do Aparelho Digestivo & 42 & 1,80 & 7,89 \\
\hline $\mathrm{X}-\mathrm{D}$. do Aparelho Geniturinário & 25 & 1,07 & 4,69 \\
\hline XII - D. da Pele e Tecido Celular Subcutâneo & 8 & 0,34 & 1,50 \\
\hline XIII - D. do Sist. Osteomuscular e do Tec. Conjuntivo & 6 & 0,26 & 1,13 \\
\hline XIV - Anomalias Congênitas & 40 & 1.72 & 7,51 \\
\hline XV - Certas causas de Morbid. e Mort. Perinatais & 3 & 0.13 & 0,56 \\
\hline XVI - Sintomas e Estados Mórbidos Mal-Definidos & 1.196 & 51.40 & 224,64 \\
\hline XVII - Acidentes, Envenenamentos e Violências & 16 & 0,69 & 3,01 \\
\hline$Y^{*}$ - Internação para Exames Diagnósticos & 5 & 0,21 & 0,94 \\
\hline Total & 2.327 & 100,00 & 437,08 \\
\hline
\end{tabular}

* Classificações Suplementares da CID.

Quando verificada a composição de cada um desses grupos de doenças, observou-se que $70,8 \%$ dos diagnósticos do Grupo 1 consistiam de diarréias, $79,6 \%$ do Grupo VIII, de pneumonias e $96,7 \%$ do Grupo XVI, de desidrataçōes.

Portanto, somente 3 causas - diarréia, desidratação e pneumonia - foram responsáveis por $80,36 \%$ đas internações de crianças menores de um ano e por $5,78 \%$ das internações de todas as idades (Tabela 4 ).
O diagnóstico de desidratação pode ser considerado conjuntamente com $O$ de doenças diarreicas, dado que representa na grande maioria dos casos, em nosso contexto sanitário, um quadro decorrente de gastro-enterites. Aliás, era o que ocorria freqüentemente com o diagnóstico de desidratação que pela regra 5 de modificação da causa básica do óbito, deveria ser preterido a favor de um diagnóstico anotado no atestado de óbito e que pertencesse a outro grupo que não o XVI (sintomas e estados mórbidos mal-definidos). 
BARROS, M.B. de A. Morbidade e mortalidade hospitalar de crianças menores de um ano, em Ribeirão Preto, SP (Brasil), 1975. Rev. Saúde públ., S. Paulo, 15:308-20, 1981.

TA B E L A 4

Diagnósticos mais frequentes das internaçōes de crianças menores de um ano, Ribeirão Preto, 1975.

\begin{tabular}{|c|c|c|c|c|}
\hline Diagnóstico & $\begin{array}{l}\text { Número de } \\
\text { internações }\end{array}$ & $\begin{array}{l}\text { Coef. } \\
(/ 1.000)\end{array}$ & $\%$ & $\begin{array}{c}\text { Indice por } 1.000 \\
\text { internaços * }\end{array}$ \\
\hline \multicolumn{5}{|l|}{ D. Diarréica } \\
\hline$(001-009) * *$ & 165 & 30,99 & 7,09 & 5,05 \\
\hline \multicolumn{5}{|l|}{ Pneumonia } \\
\hline$(480-486)$ & 549 & 103,12 & 23,59 & 16,81 \\
\hline \multicolumn{5}{|l|}{ Desidratação } \\
\hline$(788.0)$ & 1.156 & 217,13 & 49,68 & 35,40 \\
\hline Sub-total & 1.888 & 354,62 & 81,13 & 57,82 \\
\hline Demais doenças & 439 & 82,46 & 18,87 & 13,45 \\
\hline Total & 2.327 & 437,08 & 100,00 & 71,27 \\
\hline
\end{tabular}

* Internações de todas as idades da população residente em Ribeirão Preto.

** Código da CID - 8* Revisão 8.

$\mathrm{Na}$ 9a revisão da $\mathrm{CID}^{2}$ o diagnóstico de desidratação passou para o Grupo III doenças das glândulas endócrinas, da nutrição e do metabolismo e transtornos imunitários - sob código 276.5, o que deverá levar a importante aumento de freqüência deste Grupo (III) quando da aplicação da $9^{a}$ revisão.

A elevada frequiência deste diagnóstico, incorreto à medida que não inclui a causa fundamental que $o$ desencadeia, enseja a oportunidade de se alertar sobre a necessidade de anotação completa e especifica dos diagnósticos nos atestados de óbito e, em geral, nos registros de serviços médicos.

A inexpressiva presença das causas perinatais decorre da não inclusão dos recém-nascidos, neste estudo.

A ocorrência tão elevada de diarréia, desidratação e pneumonia nas hospitali- zações de crianças, em Ribeirạ̃o Preto, denota um perfil mórbido prevalente nas áreas menos desenvolvidas, e a carência alimentar e precárias condições a que estão expostos setores da população.

A intervençăo tecnicamente adequada sobre estas patologias implicaria, basicamente, no desenvolvimento de ações em outros niveis que não o hospitalar.

\section{Morbidade Hospitalar e Categoria de Internação}

As causas das hospitalizações de menores de um ano revelaram diferenciações importantes segundo a categoria de internação das crianças, como pode ser observado na Tabela 5. 
BARros, M.B. de A. Morbidade e mortalidade hospitalar de crianças menores de um ano, em Ribeirão Preto, SP (Brasil), 1975. Rev. Saúide públ., S. Paulo, 15:308-20, 1981.

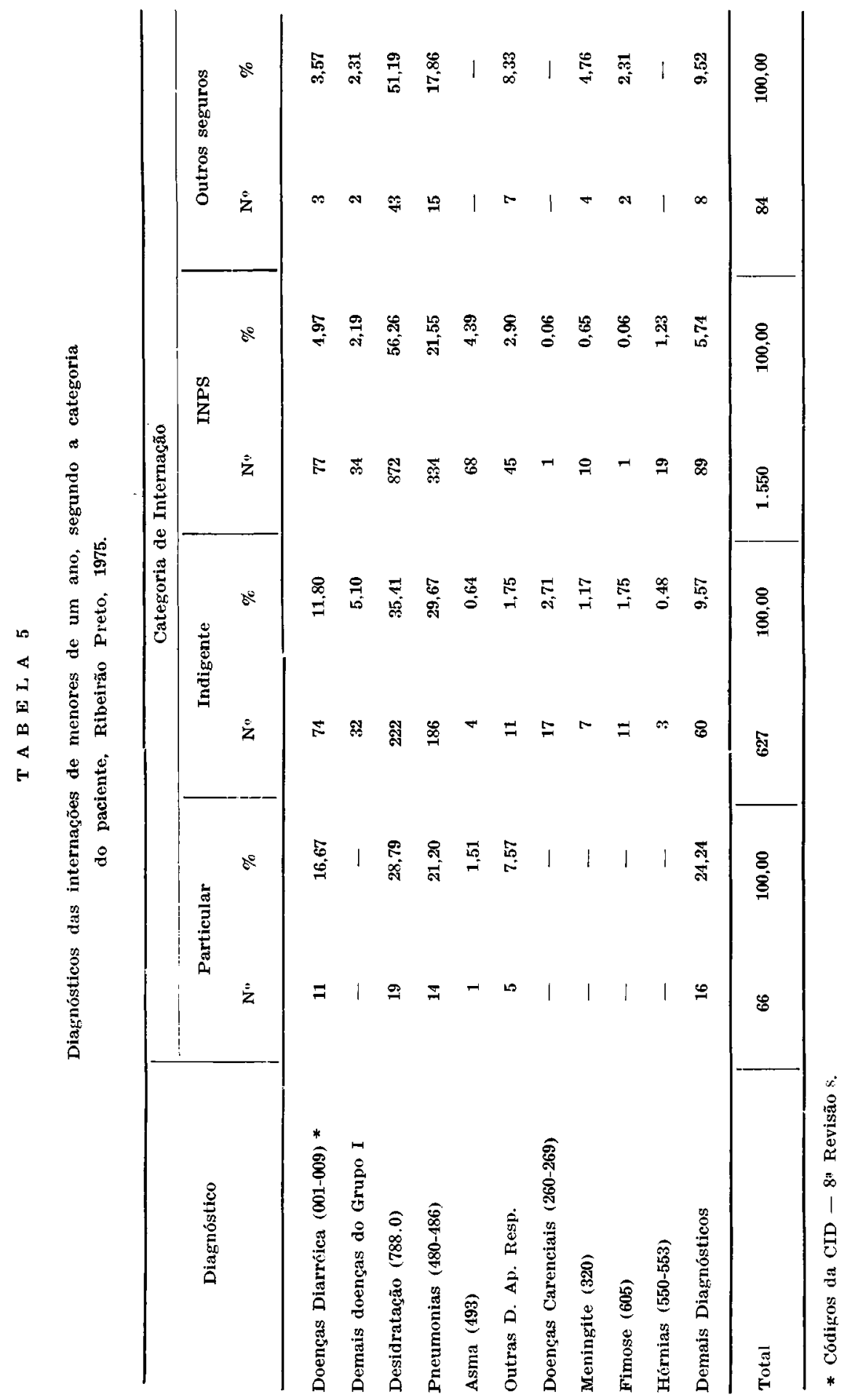


BARROS, M.B. de A. Morbidade e mortalidade hospitalar de crianças menores de um ano, em Ribeirão Preto, SP (Brasil), 1975. Rev. Saúde púlul., S. Paulo, 15:308-20, 1981.

Nas crianças da categoria "particular" estiveram ausentes as doenças carenciais, meningite e "outras doenças infecciosas", assim como foram proporcionalmente menos frequientes os diagnósticos de desidratação. Por outro lado, os "demais diagnósticos" representaram $24,0 \%$ das hospitalizações dos "particulares", não tendo atingido $10,0 \%$ nas demais categorias.

Foram as crianças indigentes as que apresentaram o maior percentual de doenças carenciais, doença diarréica, pneumonia e "outras doenças infecciosas".

Estas diferenças no quadro mórbido devem refletir condições de vida distintas destas crianças, à medida que, como referido anteriormente, às categorias de internação correspondem diferenciados estratos sócio-econômicos.

Por outro lado, algumas observações na morbidade hospitalar parecem não decorrer da relação entre categoria de internação e nível de vida, mas de peculiaridades próprias da instituição médica. Assim, a maior proporção de fimoses, entre os indigentes, poderia estar dependente do objetivo de ensino que norteia as atividades do hospital-escola, que é a instituição que lhes presta a maior parte da assistência médica.

Os maiores percentuais de hérnia, asma e desidratação verificados nas internações do INPS parecem também, decorrer mais de interesses vinculados às instituições hospitalares que prestaram os serviços, do que a um padrão de morbidade especial das crianças cobertas pela previdência.

A Tabela 6 que apresenta um detalhamento das causas componentes do Grupo I - doenças infecto-parasitárias - revela maior proporção de patologias evitáveis por vacinação e por medidas profiláticas especificas (tratamento de sifilis em gestantes) assim como das "demais doenças infecciosas", nas crianças indigentes e filhos de segurados do INPS, tendo sido poupadas destas causas as crianças da categoria particular.

\section{Mortalidade Hospitalar}

Ocorreram 63 óbitos entre as 2.327 internações de menores de um ano residentes em Ribeirão Preto, em 1975, sendo 33 do sexo masculino e 30 do feminino, o que corresponde a coeficientes de letalidade hospitalar de 3,19 e 2,32, respectivamente.

Verificou-se que pelo menos $75 \%$ dos óbitos foram devidos a doenças infecciosas e carenciais enquanto que os maiores coeficientes de letalidade hospitalar, por causa específica, foram observados para anomalias congênitas do aparelho circulatório, tumores e doenças infecciosas com exceção de diarréia (Tabela 7 ).

As crianças "indigentes" apresentaram o coeficiente de letalidade hospitalar (óbitos $\mathrm{x}$ 100/internaçōes) mais elevado, 3,16 vezes superior ao daquelas da categoria particular. Esta situação denota a persistência da desigualdade do risco de óbito, mesmo no ambiente hospitalar e apesar dos cuidados médicos de bom nível dispensados às crianças indigentes no município de Ribeirão Preto.

A razão entre os coeficientes de letalidade hospitalar de crianças "indigentes" e "particulares" $(4,94 / 1,51=3,16)$ poderia ser considerada uma estimativa mínima da razão entre os coeficientes de mortalidade infantil de setores da população com melhor e pior nível sócio-econômico.

\section{Comentários Finais}

A elevada incidência de doenças nas crianças de Ribeirão Preto fica evidenciada pela alta taxa de internação observada. A alta prevalência de morbidade na população brasileira é situação bastante conhecida ${ }^{13}$.

Entretanto, diferentemente de outros locais do pais, o município estudado conta com recursos materiais e humanos no Setor Saúde, que se situam bem acima da média nacional (1 médico para 372 habitantes e 5,25 leitos gerais/1.000 habitantes) 18 . 
BARROS, M.B. de A. Morbidade e mortalidade hospitalar de crianças menores de um ano, em Ribeirão Preto, SP (Brasil), 1975. Rev. Saúdc públ., S. Paulo, 15:308-20, 1981.

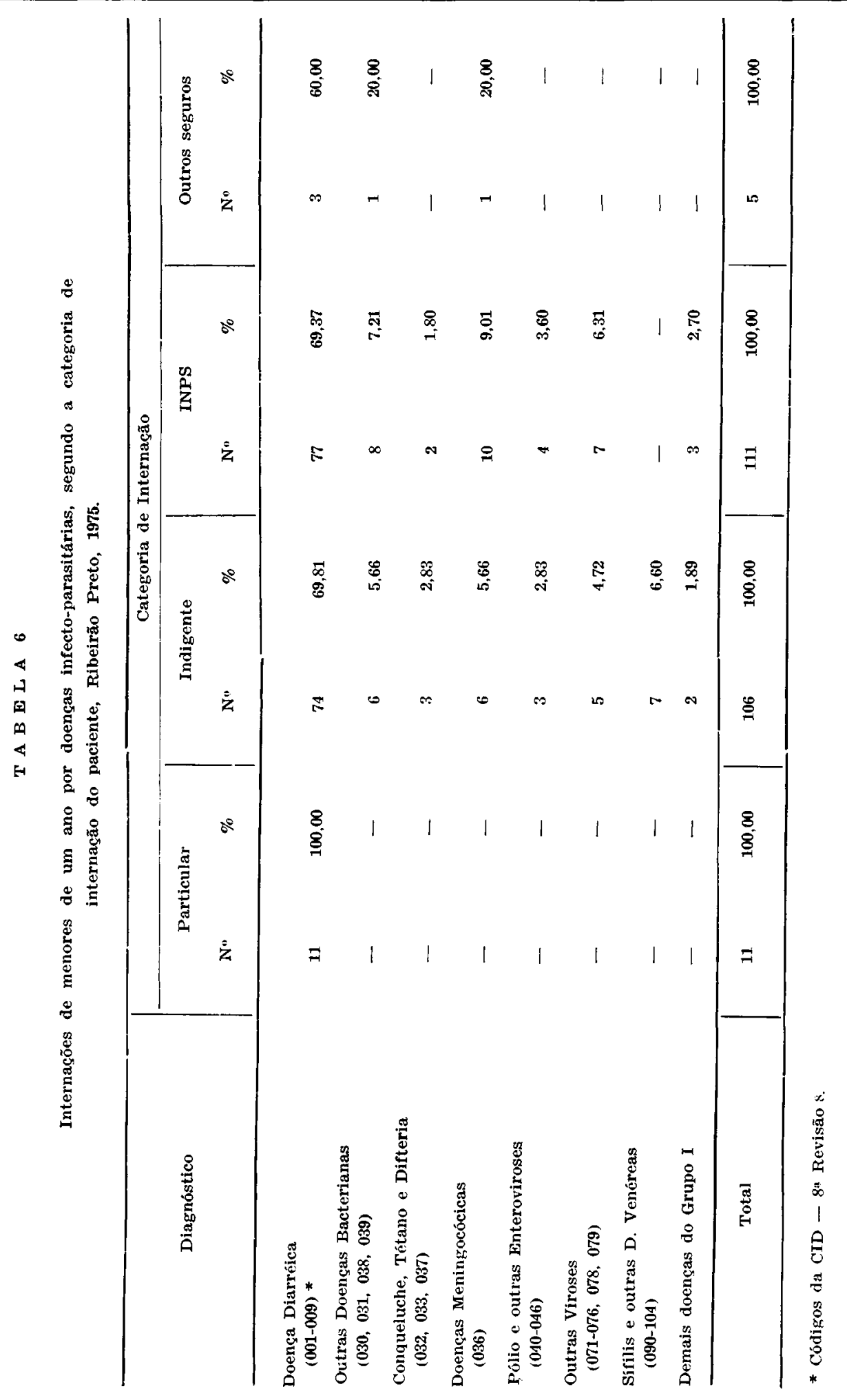


BARROS, M.B. de A. Morbidade e mortalidade hospitalar de crianças menores de um ano, em Ribeiræo Preto, SP (Brasil), 1975. Rev. Suńle públ., S. Paulo, 15:308-20, 1981.

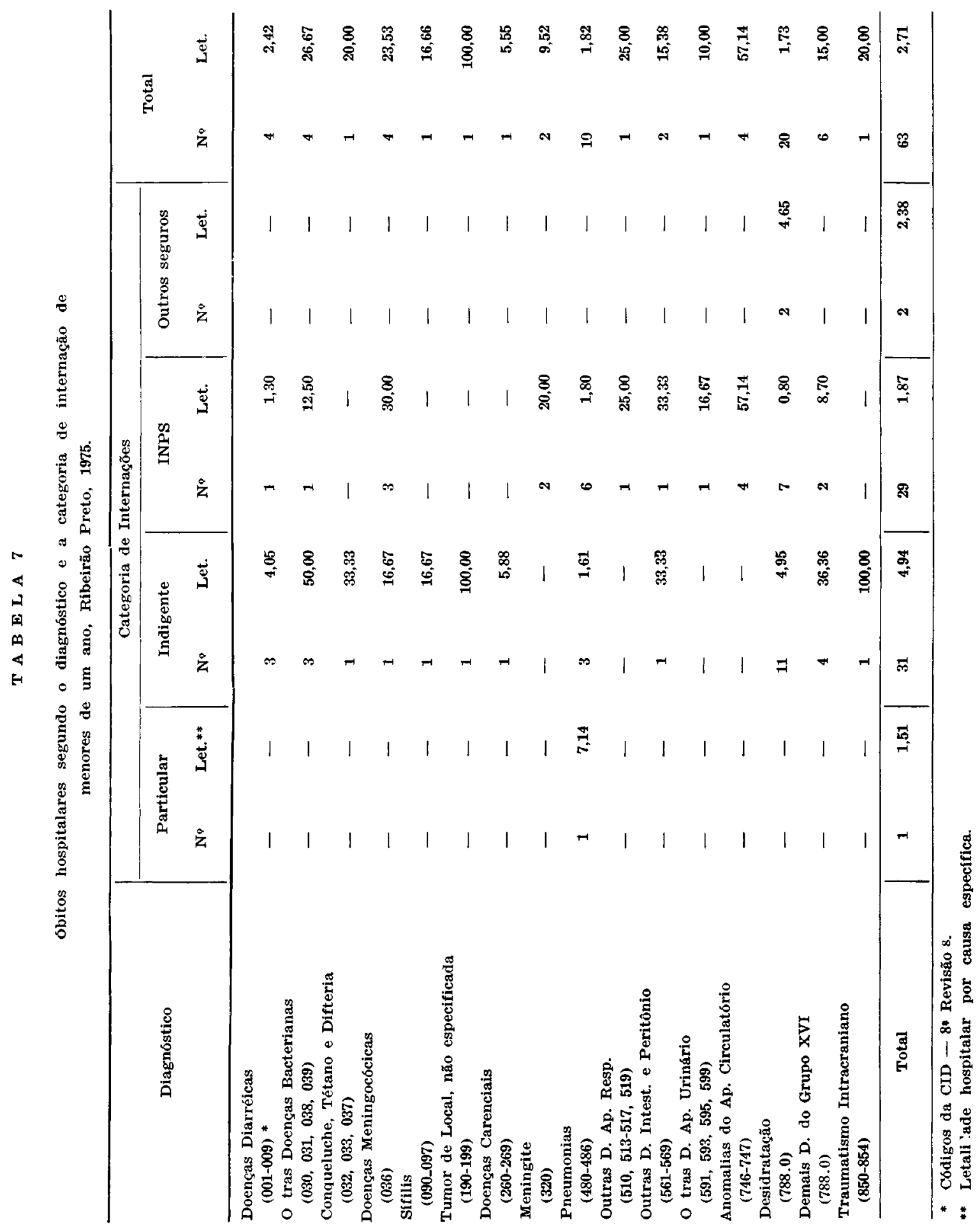


BARROS, M.B. de A. Morbidade e mortalidade hospitalar de crianças menores de um ano, em Ribeirão Preto, SP (Brasil), 1975. Rev. Saúde públ., S. Paulo, 15:308-20, 1981.

Num contexto de elevada morbidade e disponibilidade de recursos, altas taxas de utilização de serviços médicos são, então, observadas.

Quando configuradas as causas das hospitalizações, constituídas majoritariamente por diarréias e pneumonias, fica evidenciado um perfil de morbidade prevalente em áreas subdesenvolvidas e em populações carentes.

A "Investigação Interamericana de Mortalidade na Infância" ${ }^{15}$ mostrou que, no município de Ribeirão Preto, 78,1\% dos óbitos pósneonatais por diarréia e $47,1 \%$ daqueles por doenças do aparelho respiratório estavam associados à presença de desnutrição, sendo que estas percentagens foram superiores às de qualquer outra área investigada neste estudo. Como assinala Teruel ${ }^{16}$ (1971) esse percentual de associação com desnutrição é uma estimativa mínima do problema.

Os diagnósticos mais frequientes nas internações estão associados à presença de desnutrição e como "a questão de uma alimentação correta. prende-se, acima de tudo, às condições econômicas existentes" (Mello 10, 1978), estas doenças sofrem influência das políticas econômicas e da distribuição de renda.

A deficiência nutricional concentra-se em determinados setores da população, tendo sido verificado no município de São Paulo que desnutrição de II e III graus (classificação de Gomes) foram observadas apenas em crianças cuja renda familiar mensal per capita era inferior a 2,5 salários minimos, mas concentrando-se principalmente naquelas em que essa renda era inferior a 0,5 salários mínimos\%. Também no Vale do Ribeira, Monteiro ${ }^{12}$ (1979) encontrou prevalência importante de desnutrição (l e II graus) nos filhos de assalariados $(16,0 \%)$ e arrendatários (14,3\%) estando quase ausente na prole dos pequenos proprietários $(1,7 \%)$.

A distribuição preferencial destas doenças em determinados grupos sociais fica reforçada pela observação de que embora amplos setores da população de Ribeirão Preto sejam cobertos por rede pública de água $(83,0 \%)$ e de esgotos sanitários $(80,0 \%)$, as diarréias (incluidas as desidratações) persistem em elevada proporção $(57,0 \%)$ nas internações de crianças menores de um ano, residentes no município.

Outra situação que transparece neste estudo é a desarticulação existente, de um ponto de vista técnico, entre a patologia da população e o tipo de serviços disponíveis. As principais doenças detectadas nas internações são de grande vulnerabilidade e evitáveis por medidas de promoção de saúde e prevenção específica, suficientemente conhecidas. No entanto, na prevalência deste quadro mórbido, estruturam-se serviços de saúde que atuam a nível de limitação das incapacidades, portanto, bastante tardiamente. Esta forma de assistência mantém a estrutura epidemiológica prevalente, o continuo risco de doença e óbito para a população exposta e os elevados gastos hospitalares por parte da Previdência Social. A intensa produção de altas hospitalares assenta-se na lógica de um sistema de serviços médicos que privilegia o setor privado-hospitalar, e onde o setor público se adequa às normas hegemônicas enfatizando intervenções médicas em detrimento das ações de promoção da saúde.

\section{CONCLUSOES}

1. Observou-se elevado coeficiente de internação de crianças menores de um ano, no município de Ribeirão Preto, em 1975 (437/1.000); o coeficiente foi maior entre as crianças do sexo masculino.

2. As internações desta faixa etária corresponderam a $7,13 \%$ do total das hospitalizações dos residentes no município, em 1975.

3. Os principais diagnósticos de internação foram diarréia, desidratação e pneumonia, que em conjunto represen- 
BARRoS, M.B. de A. Morbidade e mortalidade hospitalar de crianças menores de um ano, em Ribeirão Preto, SP (Brasil), 1975. Rev. Saúde públ., S. Paulo, 15:308-20, 1981.

taram $80,36 \%$ das internações de crianças menores de um ano.

4. Os diagnósticos de internação apresentaram diferenciações em função da categoria de internação do paciente, que parecem ser decorrentes de diferenças sócio-econômicas existentes entre as categorias de internação e de objetivos próprios da instituiçăo que presta o cuidado médico.

5. A letalidade hospitalar é 3,16 vezes maior nas internações de crianças "indigentes" que naquelas da categoria particular, sendo que a mortalidade hospitalar decorre principalmente $(75 \%$ dos casos) de doenças infecciosas $e$ carenciais.

BARROS, M.B. de A. [Hospital morbidity and mortality among children under one year of age, Ribeirão Preto, SP (Brazil), 1975]. Rev. Saúde públ., S. Paulo, 15: $308-20,1981$.

ABSTRACT: The data of 1975 of hospital morbidity and mortality among children under one year of age was studied in Ribeirão Preto, S. Paulo, Brazil. The hospitalization rate for these children, excluding newborn, was very high - 437 per 1000 - and was higher for boys than for girls. Diarrhea, dehydration, and pneumonia accounted for $80.36 \%$ of admissions. There were, however, evident differences in morbidity related to categories of hospitalization. Infectious diseases were responsible for the largest portion (75\%) of hospital deaths among these children, and mortality was over 3 times greater for indigent children than for those whose care was remunerated.

UNITERMS: Hospital. Mortality. Morbidity. Child, hospitalized, Ribeirão Preto, SP, Brazil.

\section{REFERENCIAS BIBLIOGRÁFICAS}

1. BARROS, M.B.A. Morbidade hospitalar no municipio de Ribeirão Preto em 1975. Ribeirão Preto, 1977. [Dissertação de Mestrado - Faculdade de Medicina de Ribeirão Preto da USP].

2. BATISTA. M. Prevaléncia e estágio da desnutriçāo protéico-calorica em crianças da cidade de São Paulo. São Paulo, 1976. [Tese de Doutoramento - Escola Paulista de Medicina].

3. FAVERO, M. et al. Organização de um centro de informática hospitalar em nível local. Rev. paul. Hosp., 21:151-7, 1973.

4. GLICKMAN, L. Inpatient utilization of short-stay hospitals by diagnosis. Vital Hlth Statist. Serie 13, (35) 1978.

5. KOHN, R. \& WHITE, K.L. Health care: an intemational study; Report of the World Health Organization/International Collaborative Study of Medical Care Utilization. London, Oxford University Press, 1976.
6. LANDMANN, J. Saúde e assistência médica: determinantes. Rev. Div. nac. Pneumol. sanit., $22(85 / 86): 55-84,1978$.

7. LAURENTI, R. \& PASTORELO, E.F. A mensuração das condições de saúde nas comunidades. In: Pareta, J.M.M. et al., Saude e comunidade: temas de medicina preventiva e social. São Paulo, McGraw-Hill do Brasil, 1976. p. 33-59.

8. MANUAL da Classificação Estatística Internacional de Doenças, Lesões e Causas de obito. Washington, D.C., Organização Panamericana da Saúde, 1969. (OPAS Publ. Cient., 190).

9. MANUAL da Classificação Estatística Internacional de Doenças, Lesões e Causas de óbito; revisão, 1975. São Paulo, Centro da OMS para Classificação de Doenças em Português, 1978. 
BARROS, M.B. de A. Morbidade e mortalidade hospitalar de crianças menores de um ano, em Ribeirão Preto, SP (Brasil), 1975. Rev. Saúde públ., S. Paulo, 15:308-20, 1981.

10. MELO, Z.F, A reprodução da força de trabalho e os indices salariais. Encontros com a Civilizacão Brasileira, Rio de Janeiro, $2: 123-37,1978$.

11. MONETTI, V. \& CARVALHO, P.R. Mortalidade materna e na infancia no Estado de São Paulo: aspectos demograficos, sócio-culturais e médico-sanitários. 2" ed. São Paulo, Secretaria da Saúde do Estado de São Paulo. Instituto de Saúde, 1976. (Publ., 29).

12. MONTEIRO, C.A. Os determinantes da desnutrição infantil no Vale do Ribeira. Cad. Pesq., S. Paulo, 29:57-75. 1979.

13. NORONHA, J.C. \& GUIMARAES, R. As atuais conđições de saúde da população e seus determinantes. In: Guimarães, $R$. Saúde e medicina no Brasil: contribuigão para um debate. Rio de Janeiro, Graal, 1978. p. 31-43.

14. PEARSON, J.C. et al. Hospital caseloads in Liverpool, New England and Uppsala. Lancet, 2:559-66, 1968.
15. PUFFER, R.R. \& SERRANO, C.V. Caracteristicas de la mortalidad en la nẫez. Washington, D.C., Organizacion Panamericana de la Salud, 1973. (OPAS - Publ. Cient., 262).

16. TERUEL, J.R. Mortalidade por diarreia c desnutrigão na infancia. Ribeirăo Preto. 1971. [Tese de Livre-Docência - Faculdade de Medicina de Ribeiráo Preto da USP].

17. YAZLLE ROCHA, J.S. Morbidade e mortalidade hospitalar em Ribeirão Preto, SP, Brasil, 1972. Rev. saúde públ., S. Paulo, $11: 214-28,1977$.

18. YAZLLE ROCHA, J.S. Utilização de leitos hospitalares gerais em Ribeirão Preto, São Paulo (Brasil). Rev. Saúde públ., S. Paulo, 9:477-93, 1975 .

Recebido para publicasão em 18/11/1980 Aprovado para publicą̧a em 08/18/1980 